JOANNA GORECKA-KALITA

Université Jagellonne

e-mail: joanna.gorecka.kalita@gmail.com

\title{
La « laide vérité ». Le rêve de Cahus revisité
}

\author{
Abstract \\ "The Ugly Truth". Cahus' Dream Revisited
}

The aim of this paper is to propose an analysis of the Cahus' Dream, a well known episode of the Perlesvaus, Arthurian romance from the $13^{\text {th }}$ century, within the context of the medieval dream theories. Inspired mostly by Macrobius' Commentary on the Dream of Scipio - focusing on the divinatory (or deceptive/ illusory) role of dreams - as well as by Tertullian's and Augustine's Christian reflections on the relations between the soul and the sleeping body, these theories permit to shed a new light on the oneiric adventure of the squire. In fact, the author furnishes numerous clues which make it look as an insomnium or fantasma: a false, illusory dream, deprived of any deeper signification. Thus, unable of uncovering some hidden, symbolic meaning, the mirage paradoxically turns out to be a material, "ugly", as the text has it, truth, blurring the border between dream and reality in a most confusing way, and setting the specific Perlesvaus tone from the very beginning of the romance.

Keywords: Arthurian romance, Perlesvaus (Le Haut Livre du Graal), dream turning real, nightmare, phantasm, medieval dream theories, Macrobius, Augustine, divination

Le Perlesvaus ${ }^{1}$, cet « étrange monstre » arthurien du XIII ${ }^{\mathrm{e}}$ siècle - roman paradoxal, flamboyant, « livre de haute violence $»^{2}$, " soulevé par l'Esprit de la Révélation et hanté par la fantasmagorie celtique $»^{3}-$ ne cesse d'interpeller les critiques. Ainsi, l'épisode du rêve de Cahus - l'un des plus étranges du roman -

1 Il existe deux éditions du roman : celle de W.A. Nitze et Th. A. Jenkins (Le Haut Livre du Graal [Perlesvaus], New York 1972), et celle, plus récente, d' A. Strubel, accompagnée d'une traduction (Le Haut Livre du Graal [Perlesvaus], Paris 2007). Les citations dans l'article proviennent de cette dernière édition.

2 F. Dubost, Le Perlesvaus, livre de haute violence, « Senefiance» 1994, no. 36 (La violence dans le monde médiéval), p. 180-199.

3 Ch. Méla, La reine et le Graal. La conjointure dans les romans du Graal, de Chrétien de Troyes au Livre de Lancelot, Paris 1984, p. 182. 
a suscité pour sa part un bon nombre d'interprétations ${ }^{4}$, allant de lectures « traditionnelles » jusqu'aux plus (post)modernes : psychanalytique, fantastique, herméneutique, lacanienne... Celle que j'entends proposer ici vise à placer ce récit dans le contexte des théories médiévales du rêve : cette approche, en apparence peu novatrice, permet pourtant, je l'espère, d'y jeter un éclairage nouveau.

L'épisode de Cahus est situé au début du roman ${ }^{5}$. Après un prologue ressemblant à un évangile apocryphe, alléguant Dieu Lui-même comme Auteur suprême du livre, le narrateur décrit une situation de crise : le roi Arthur est en proie à une « volentez delaianz », une sorte d'acédie qui lui fait « perdre le talent » de faire le bien et lui fait abandonner les pratiques religieuses. Un jour de l'Ascension il décide, sur l'incitation de la reine Guenièvre, d'entreprendre un pèlerinage à la mystérieuse chapelle Saint-Augustin. Toujours suivant les conseils de son épouse, il accepte - malgré de mauvais pressentiments - de prendre comme compagnon un jeune écuyer nommé Cahus.

À la tombée du soir, Cahus se couche seul dans la salle : « Il ne se voust desvetir ne deschaucier, car la nuiz li sambloit estre corte, et por ce qu'il voloit estre prez au matin au commandement le roi. (...) E o premerain somme qu'il fu endormiz, li sanbloit que li rois en ert allé sanz lui ». S'ensuit le récit du rêve: Cahus, « molt effreez », enfourche son cheval et se met à la poursuite du roi. Son chemin onirique le conduit au fond d'une grande forêt, jusqu'à une chapelle entourée d' " un grant cimetiere ou il avoit molt sarqeux ». L'écuyer pénètre dans la chapelle où gît un chevalier mort, entouré de quatre cierges brûlant dans quatre chandeliers d'or. Cahus s'empare d'un des chandeliers - le narrateur n'explique nullement le mobile de cette action -, l'enfile dans sa chausse, et quitte la chapelle. Sur son chemin de retour il aperçoit soudain « un home noir et let, [qui] estoit assez graindres a pié que cil n'estoit a cheval, e tenoit .i. grant cotel agu en sa main, a .ii. trenchanz, ce li estoit avis ». Celui-ci revendique le chandelier, et lorsque Cahus tente de l'esquiver, l'assaillant « le fiert du cotel o destre costé si qu'il li enbat o cors desq'enz manche ». À ce moment-là le jeune homme - « qui gisoit en la sale a Carduell, qi ce ot songié », précise le narrateur - se réveille avec un cri déchirant : " Sainte Marie ! le provoire ! Aidiez, aidiez, car ge sui morz ! » Lorsqu'accourent le roi et les chambellans, il leur fait le récit de son aventure. « A ! fet li rois, est-ce dont songes ? - Oïl, sire, fet il. Mes il m'est molt ledement averez »: Cahus exhibe alors le couteau planté dans son côté gauche et sort de sa chausse le chandelier d'or. Après que l'écuyer se confesse, le roi retire le couteau

${ }^{4}$ Pour ne citer que les plus importantes : A.H. Krappe, Balor with the Evil Eye, Studies in Celtic and French Literature, New York 1927 ; M. Williamson, The Dream of Cahus in the "Perlesvaus ", « Modern Philology » 1932, Aug., vol. 30, no. 1, p. 5-11 ; Ch. Méla, op. cit. ; M. Zink, Le rêve avéré. La mort de Cahus et la langueur d'Arthur du Perlesvaus à Fouke le Fitz Waryn [dans :] Mélanges René Fromilhague, éd. Cl. Sicard, Université de Toulouse-Le Mirail, Toulouse 1984, p. 31-37 ; F. Dubost, Aspects fantastiques de la littérature narrative médiévale (XII ${ }^{e}-X I I I^{e}$ siècles). L'Autre, l'Ailleurs, l'Autrefois, Paris 1991 ; A.M. Williams, Dreams and Visions in the Perlesvaus [dans :] B. Wheeler, Arthurian Studies in Honour of P.J.C. Field, Cambridge 2004 ; B. Ramm, A Discourse for the Holy Grail in Old French Romance, Cambridge 2007.

5 Pages 136-140 de l'édition de Strubel. En raison de la brièvete de l'épisode, je n'indique pas de numéro de page pour les citations. 
de son corps et «l'enme s'en parti lués ». Le roi, après l'avoir richement enseveli, part seul vers l'ermitage ; le chandelier sera offert à l'église Saint-Paul de Londres - « li rois voloit que cele aventure mervelleuse fust seüe par tot, et que on priast en l'eglise por l'ame au vallet qui por chandellabre fu ocis »- et il n'en sera plus question dans le texte.

De nombreux critiques ont essayé d'élucider le sens caché, de dévoiler quelque « vérité coverte » sous la « semblance » du rêve. Or, l'attitude médiévale face aux rêves est marquée, on le sait, par une profonde ambivalence. Celle-ci concerne deux aspects, respectivement celui du songe et celui du sommeil : il s'agit premièremement de la valeur herméneutique des rêves, et deuxièmement, du statut du dormeur, de ce que devient son âme et son corps pendant le sommeil. Il me semble donc nécessaire ici de faire un détour et de résumer les grandes lignes des théories onirologiques du Moyen Âge, pour situer ensuite le rêve de Cahus sur ce fond.

En ce qui concerne le premier aspect du songe, le Moyen Âge fait sien, d'une part, l'héritage de la culture gréco-romaine, où les rêves étaient traités comme une source du savoir sur les événements à venir - savoir parfois illusoire, mais en général véridique -, et d'autre part l'héritage patristique, où le songe peut être l'espace d'une révélation, mais plus souvent se révèle être une illusion diabolique $^{6}$. La réflexion chrétienne s'accompagne donc dès le début d'une méfiance (croissante au fil du temps) à l'égard du monde onirique. En même temps, dans leur classement des songes, les théologiens et les encyclopédistes médiévaux reprennent, mutatis mutandis, les théories antiques : le Commentaire au songe de Scipion du néo-platonicien Macrobe est bien l'un des textes les plus lus par les intellectuels du XII ${ }^{e}$ siècle. Or, dans sa typologie, Macrobe - reprenant lui-même une classification dont il souligne l'ancienneté ${ }^{7}$ - divise l'ensemble des images oniriques en cinq catégories :

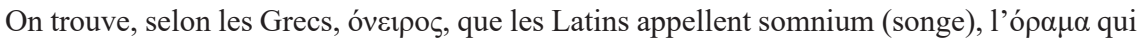
est à proprement parler la uisio (vision), le $\chi \rho \eta \mu \alpha \tau \imath \sigma \mu o ́ \varsigma$ que l'on nomme oraculum (oracle), l'Evvлviov ou insomnium (vision interne au songe), le $\varphi \alpha ́ v \tau \alpha \sigma \mu \alpha$ que Cicéron [...] a traduit par uisum (fantasme).

Les deux derniers, quand ils se manifestent, ne valent pas la peine qu'on les interprète, parce qu'ils ne fournissent aucun élément divinatoire ${ }^{8}$.

Ainsi Macrobe, de même que les autres auteurs antiques ${ }^{9}$, oppose les rêves « vrais » aux rêves trompeurs. Et c'est bien la définition qu'il propose de ces derniers qui me semble particulièrement intéressante dans le contexte de l'épisode de Cahus :

Il y a en effet $\varepsilon v v \pi v 10 v$ quand une préoccupation oppressante d'origine psychique, physique ou extérieure s'offre au dormeur sous la même forme dont elle l'obsédait, éveillé (...). Ces

6 V. J. Le Goff, Le christianisme et les rêves [dans :] L’imaginaire médiéval, Paris 1985, p. 263-330.

7 Macrobe, Commenaire au Songe de Scipion, livre I, éd. M. Armisen-Marchetti, Paris 2001, I, 3, 1-2.

8 Ibid., I, 3, 2-5.

9 V. H. Braet, Le songe dans la chanson de geste, «Romanica Gandensia » 1975, no. 15, ch. I : « La théorie du rêve selon les anciens et les pères », p. 17. 
représentations et autres semblables, qui ont perturbé le repos du dormeur de la même façon qu'elles l'avaient précédé, proviennent d'une disposition de l'âme ; aussi s'envolent-elles en même temps que le sommeil et disparaissent avec lui. D'où le nom d'insomnium : (...) parce qu'on n'y accorde crédit que durant le songe même, aussi longtemps qu'on l'a sous les yeux : après le songe il ne présente plus d'intérêt ni de signification ${ }^{10}$.

Cette catégorie des rêves émanant de l'homme - distincts aussi bien de l'illusion diabolique que de la révélation mystique - se retrouve sous la plume de Grégoire le Grand ${ }^{11}$, et, quelques siècles plus tard, chez Honoré d'Autun qui, répondant à la question sur l'origine des rêves, donne l'exemple de l'homme « qui voit en son songe l'image de ce qu'il a vu, entendu, ou ce à quoi il a pensé à l'état de veille $»^{12}$.

Or, il est évident que le rêve de Cahus reproduit assez fidèlement ses préoccupations réelles : il a si peur d'être en retard, qu'il se couche tout habillé dans la grande salle du château. Ainsi, son rêve - qui débute justement avec ce retard tant appréhendé ( « li sanbloit que li rois en ert allé sanz lui ») et la poursuite effrénée du roi ( « li estoit avis en sonjant qu'il issoit du chastel grant aleüre aprés le roi Artu») apparaît comme une prolongation naturelle de la pensée qui l'obsédait à l'état de

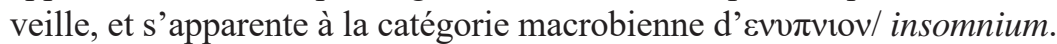

Qui plus est, l'auteur précise que le rêve de Cahus a lieu - ce à quoi il me semble que l'on n'a pas suffisamment attiré l'attention - « o premerain somme ». Voilà ce qu'en dit Macrobe dans la suite de sa description des rêves trompeurs :

Quant au $\varphi \alpha ́ v \tau \alpha \sigma \mu \alpha$, c'est à dire au uisum, il se produit entre veille et repos profond, dans cette espèce, comme on dit, de première brume du sommeil (prima somni nebula), quand le dormeur, qui se croit encore éveillé alors qu'il commence tout juste à sommeiller, rêve qu'il aperçoit, fondant sur lui ou errant ça et là, des silhouettes qui diffèrent des créatures

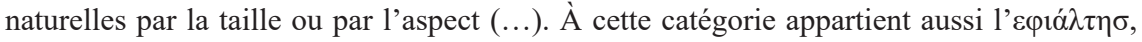
qui selon la croyance populaire s'empare des dormeurs et, pesant sur eux de tout son poids, les écrase de façon perceptible ${ }^{13}$.

Le rêve de Cahus serait donc non seulement influencé, voire provoqué directement par les préoccupations diurnes, mais aussi peuplé par les hallucinations inconsistantes, inhérentes à cette première phase du sommeil qu'Alfred Maury nommera, bien des siècles plus tard, l'état hypnagogique ${ }^{14}$. Ainsi, le " géant au couteau " semble appartenir a à ce type de figures hallucinatoires décrites par Macrobe, tandis que l'attaque et la blessure rapprochent ce songe de l' $\varepsilon \varphi t \alpha ́ \lambda \tau \eta \sigma$.

Or, " ces deux types de songes ne sont d'aucun secours pour connaitre le futur $»^{15}$, déclare Macrobe. Les auteurs médiévaux - depuis Grégoire le Grand

10 Ibid., I, 3, 1-5.

11 Grégoire le Grand, Dialogues, PL (Patrologia Latina) 77, col. 409B-411A.

12 Honoré d'Autun (Honorius Augustodunensis), Elucidarium sive dialogus de summa totius christianae theologiae, PL 172, col. 1163A : «Aliquando ab ipso homine, cum quod viderit, vel audierit, vel cogitaverit, hoc in somnis imaginatur».

13 Macrobe, op. cit., I, 3, 7 (p. 12).

14 A. Maury, Des hallucinations hypnagogiques ou des erreurs des sens dans l'état intermédiaire entre la veille et le sommeil, « Annales médico-psychologiques » 1848, I, p. 26-40.

15 Macrobe, op. cit., I, 3, 8 (p. 12). 
jusqu'à Adélard de Bath et l'auteur de Somnia Danielis ${ }^{16}$ - partagent cette croyance ancienne qui, comme l'écrit Hermann Braet, « veut que les songes faux se produisent dans le premier sommeil et les songes véridiques après minuit $»^{17}$. Ainsi, de nombreux indices disposés dans le texte - la mention du premier sommeil, le lien avec les préoccupations réelles, le contenu et l'articulation des images oniriques - semblent suggérer que le rêve de Cahus n'est qu'un fantasme dépourvu de toute signification. En témoigne aussi la réaction du roi : la question « A ! estce dont songes? » semble minimiser l'importance du récit qu'il vient d'entendre. Par conséquent, l' " averement » du rêve éclate de manière saisissante dans toute sa sanglante brutalité, avec le couteau fiché « o cors desqu' au manche» et la mort qui en résulte.

Aucune « senefiance » du rêve ne sera donnée dans la suite du roman, excepté les paroles laconiques du roi qui l'avance à la reine comme une preuve du bienfondé de ses pressentiments antérieurs : «Vos poez bien savoir, par cele aventure qui avenue est, que Dex ne volt consentir que nus voist avec moi ». Il est difficile d'accepter cette explication : l'on voit mal, en effet, Dieu se servir d'une créature démoniaque pour éliminer le compagnon du roi, et tout ceci dans l'espace d'un cauchemar $^{18}$ ! Mais c'est bien là une confusion typiquement perlesvausienne : le roman présente en effet plusieurs situations qui baignent dans une incertitude ontologique et axiologique, et où la frontière entre le chrétien et le démoniaque s'estompe ${ }^{19}$.

Reste pourtant l'irréductible « averement » du songe, distinct de toute signification symbolique possible, se posant comme un défi à l'entendement. Le paradoxe veut que le rêve de Cahus - présenté, comme j'ai tenté de le démontrer, comme trompeur - devienne vérité à défaut d'en dévoiler une : ainsi, au lieu d'une vérité « coverte », herméneutique, le lecteur obtient une vérité « aperte », matérielle et « lede ». Ceci est tout à fait exceptionnel. Si, dans la littérature médiévale, des rêves prémonitoires ou symboliques abondent malgré de nombreuses mises en garde ecclésiastiques, la vision qu'ils véhiculent n'est que « semblance» : elle peut se réaliser par la suite - comme c'est le cas des songes épiques analysés par Hermann Braet, ou des songes émaillant le Lancelot en prose et la Mort le roi Artu - mais ne se matérialise jamais de façon aussi directe ${ }^{20}$. Les voyages oni-

16 Adélard de Bath, De eodem et diverso, éd. H. Willner, Münster 1903, p. 13 ; Somnia Danielis reproduit par M. Hélin, La Clef des songes, cité par H. Braet, op. cit., p. 25, no. 1.

17 H. Braet, op. cit., p. 24; v. aussi J. Verdon, Dormir au Moyen Âge, [dans :] Revue belge de philologie et d'histoire, t. 72, f. 4, 1994, p. 749-759 (756), idem, La nuit au Moyen Âge, Paris 1998, p. 212-213.

18 V. F. Dubost, Aspects..., p. 789.

19 Voir à ce propos mon article Les diaboliques du Perlesvaus ou les fantasmes de castration, « Romanica Cracoviensia » 2015, no. 15, p. 260-269, et J.-R. Valette, Barbarie et fantasmagorie dans Perlesvaus, le Haut Livre du Graal [dans :] Mélanges barbares. Hommage à Pierre Michel, éd. J.-Y. Debreuille, Ph. Régnier, Lyon 2001, p. 23-33.

20 A.H. Krappe, qui a consacré un chapitre de son livre Balor with the Evil Eye à l'épisode de Cahus, reconnaît le caractère exceptionnel de l'aventure, et y voit une fusion de deux motifs attestés dans le folklore : "The episode of the Squire's Adventure is the skillful fusion of two tales existing independently, the Spectres' Mass, known all over Europe, and the Dream Journey to the Fairy Land, a typically Celtic story » (A.H. Krappe, op. cit., p. 125). À la même époque, M. Williamson se montre 
riques peuvent affecter l'âme, mais non le corps : les théoriciens chrétiens du rêve sont ici unanimes. Le premier « onirologue » chrétien, Tertullien, définissant les songes comme « les accidents du sommeil et les élans non médiocres de l'âme » (accidentes somni et non modicis iactationes animae), sépare clairement le repos du corps de l'activité de l'âme :

Ainsi, lorsque le repos arrive pour les corps (...), l'âme, dédaignant un soulagement qui lui est étranger, ne se repose pas, et si le ministère des membres corporels lui manque, elle se sert des siens. Figure-toi un gladiateur sans armes, ou un cocher sans char, reproduisant, par leurs gestes, les habitudes et les efforts de leur art (...)! Toute cette agitation est vaine ; il leur semble néanmoins qu'elle est vraie, quoiqu'elle n'ait rien de vrai ${ }^{21}$.

Saint Augustin parle de « la célérité et la facilité avec laquelle l'âme fabrique en elle même l'image des corps qu'elle a vus avec les yeux du corps », mais, ajoute-t-il, « quelle que soit la nature de ses visions, il est hors de doute qu'elles ne sont point de corps $\gg{ }^{22}$. Pour réfuter la croyance en métamorphoses et voyages oniriques, profondément enracinée dans le monde antique, l'évêque d'Hippone qui reconnaît bien l'existence de songes « vrais », au sens de leur signification ${ }^{23}$ forge dans La cité de Dieu le concept de phantasticum hominis. Ainsi, explique-til, les démons sont capables de produire une image illusoire de l'homme, mais ne peuvent en aucune manière la doter de réalité corporelle :

Assurément, les démons ne sont pas créateurs de natures (...); ils modifient quant à l'apparence seulement les créatures du vrai Dieu pour qu'elles semblent être ce qu'elles ne sont pas. (...) Je crois que l'image fantomatique de l'homme, qui (...), sans être corps, prend cependant avec une rapidité étonnante les formes ressemblantes des corps, peut se présenter, quand les sens corporels de l'homme sont assoupis ou abattus, aux sens d'autres hommes sous une apparence corporelle $\mathrm{e}^{24}$.

Cette conviction domine toujours dans le haut Moyen Âge. En 906, Réginon de Prüm, dans le canon Episcopi, tranche la question des « hordes sauvages » ou « chevauchées de Diane » : celles-ci, en fait, ne relèvent que d'une fausse opinion

plus sévère à l'égard de l'auteur du Perlesvaus : « Apparently the author of Perlesvaus remembered, or had at hand, this story from Celtic tradition; but did not understand it or know how to handle it, with the confusing result that the incident as he tells it is partly a dream and partly real » (M. Williamson, op. cit., p. 11).

${ }^{21}$ Tertullien, De anima, trad. E.-A. de Genoude, Paris 1852, éditon numérisée : http://www. tertullian.org/french/french.htm (accès: 30.01.2018).

22 Saint Augustin, De Genesi ad litteram, PL 34, col. 469-470, livre XII (« De visionum causis »), ch. 18 : «Ego vero multo amplius admiror, multoque maxime stupeo quanta celeritate ac facilitate in se anima fabricetur imagines corporum, quae per corporis oculos viderit, quam somniantium vel etiam in ecstasi visiones. Quaecumque tamen illa natura visorum est, procul dubio corpus non est. » Traduction française de Charpentier, Euvres complètes de saint Augustin, t. VII, Paris 1873, ouvrage numérisé sur le site de Gallica : http://gallica.bnf.fr/ark:/12148/bpt6k65778379(accès : le 31. 01.2018). V. aussi idem, De quantiate animae, PL 32, col. 1074, ch. XXXIII, 71.

23 V. Saint Augustin, De Genesi ad litteram... : « Ego visa ista omnia visis comparo somniantium. Sicut enim aliquando et haec falsa, aliquando autem vera sunt, aliquando perturbata, aliquando tranquilla; ipsa autem vera, aliquando futuris omnino similia, vel aperte dicta, aliquando obscuris significationibus et quasi figuratis locutionibus praenuntiata: sic etiam illa omnia ».

${ }^{24}$ Saint Augustin, La cité de Dieu, livres XV-XVIII, trad. G. Combès, Paris, 1960, XVIII, XVIII, p. 537-539. 
et n’ont aucune réalité tangible ${ }^{25}$. Dans son pénitentiel Corrector sive medicus, Burchard de Worms le formule ainsi, en s'adressant à la pénitente :

As-tu partagé la croyance de nombreuses femmes, de la suite de Satan ? Que pendant le silence de la nuit, après t'être étendue dans ton lit et pendant que ton mari repose sur ton sein, tu as le pouvoir, toute corporelle que tu es, de sortir par la porte fermée, de parcourir l'espace avec d'autres femmes qui te ressemblent ? ${ }^{26}$

Ces « chevauchées », qui deviendront articles d'accusation dans les procès de sorcellerie de la fin du Moyen Âge et de la Renaissance, ne sont pour l'évêque de Worms que des superstitions qu'il faut éradiquer par le jeûne et la pénitence.

Ce qui se passe lors du sommeil peut donc avoir une influence sur l'âme : la vision, béatifique ou terrifiante, peut amener la conversion ou affermir la foi, tandis que le songe faux peut (bien que le cas soit plus rare) conduire à l'hérésie ${ }^{27}$. Mais les aventures survenues dans le cadre du songe, bien qu'elles provoquent parfois une intense douleur physique, restent en général sans effet pour le $\operatorname{corps}^{28}$.

La « laide vérité » du rêve de Cahus reste donc une énigme. L'auteur du roman, loin d'en donner la solution, brouille encore les pistes et construit consciemment une zone d'ombre autour de l'aventure. Il souligne, par exemple, la solitude du dormeur : fait inhabituel dans les chambres à coucher médiévales, où seuls les rideaux du lit délimitent parfois une zone privée. Ainsi, il n'y a aucun témoin qui pourrait objectiver l'expérience de Cahus et éclairer le mystère : d'où vient le couteau fiché dans le corps du jeune homme ? Cahus a-t-il été frappé dans son rêve, ou, victime de somnambulisme, est-il sorti du château sans être aperçu, pour vivre réellement l'aventure qu'il croit avoir rêvée ? Le phénomène de ces déambulations nocturnes - lesquelles, d'ailleurs, se produisent en général lors du premier tiers de la nuit ${ }^{29}$ - était familier aux auteurs médiévaux qui débattaient

25 Réginon de Prüm, De ecclesiasticis disciplinis, PL 132, col. 352B-352C: « Illud etiam non omittendum, quod quaedam sceleratae mulieres retro post Satanam conversae, daemonum illusionibus et phantasmatibus seductae, credunt se et profitentur nocturnis horis cum Diana paganorum dea et innumera multitudine mulierum equitare super quasdam bestias, et multa terrarum spatia intempestae noctis silentio pertransire (...). Nam innumera multitudo, hac falsa opinione decepta, haec vera esse credit ».

26 Burchard de Worms, Corrector sive medicus [dans :] C. Vogel, Le pécheur et la pénitence au Moyen Âge, Paris 1969, s. 107-108.

27 Un topos du « rêve correcteur » dans le milieu monastique est la flagellation ou une autre punition imposée pour la lecture des auteurs païens ; pour les exemples a contrario, Raoul Glaber relate deux histoires où le rêve conduit à l'hérésie (v. J.-Y. Tilliette, Belles-lettres et mauvais rêves [dans :] Le rêve médiéval, éd. A. Corbellari, J.-Y. Tillette, Genève 2007, p. 11-36).

28 Il faut toutefois noter la présence de deux récits patristiques où la flagellation subie en songe laisse des traces matérielles au réveil : saint Augustin relate le songe de son concitoyen Tutelymène, dont le dos porte les marques de coups reçus (Sermo CCCVIII, PL 38, col. 1410), et saint Jérôme raconte sa propre expérience dans la fameuse Lettre 22 à Eustochium : fustigé pour avoir lu Cicéron et Plaute, il s'en ressent douloureusement au réveil ; ces marques, pourtant, sont pour lui une preuve qu'il s'agissait là d'une vision, et non d'un songe ( « Nec vero sopor ille fuerat, aut vana somnia, quibus saepe deludimur. (...) Liventes fateor habuisse me scapulas, plagas sensisse post somnum », Saint Jérôme, Ad Eustochium, PL 22, col. 417). Ces récits ne peuvent en aucun cas élucider le sens du rêve de Cahus : leur signification chrétienne est évidente, et ils se terminent par la correction de leur héros et non pas par sa mort.

29 J. Verdon, La nuit au Moyen Âge..., p. 207. 
sur la question de la responsabilité du somnambule : au XIV siècle, dans le canon Si furiosus, le dormiens sera jugé irresponsable de l'homicide, à l'instar du fou furieux ou de l'enfant ${ }^{30}$. Cette hypothèse pourrait être appuyée par deux objets matériels qui sembleraient renvoyer vers quelque événement réel : le chandelier et le couteau. Ce deuxième artéfact, pourtant, pose problème et met fortement en cause l'explication « rationelle »: Cahus, dans son rêve, a été frappé au côté droit, mais au réveil le couteau est fiché dans son côté gauche. Les deux informations sont séparées par à peine une dizaine de vers, l'on ne peut donc l'attribuer à une erreur du copiste (d'autant plus que la même leçon se retrouve dans tous les manuscrits) $)^{31}:$ « e cil le haste, e le fiert du cotel o destre costé si qu'il li enbat au cors desqu'enz o manche $»$.

Il hauça le brez senestre. « Sire, esgardez ça. Vez ci le cotel qui m’est o cors desqu'au manche.

« Surprenant au premier abord », écrit Francis Dubost, « cette inversion apparaît à la réflexion comme le détail significatif qui spécularise le rapport du rêve à la réalité ${ }^{32}$. Le rêve serait-il donc une « seconde vie » de l'autre côté du speculum où tout s'inverse ? Cahus serait-il passé « de l'autre côté du miroir »? Le narrateur insiste à plusieurs reprises sur le caractère onirique, donc subjectif, de l'aventure (« li sembloit », « e li estoit avis en sonjant», « il se pensa en sonjant», « li vallez, qui gisoit en la sale a Carduell, qi ce ot songié » etc.) : on retrouve là la formule latine topique visus est videre ${ }^{33}$. Le candélabre et le couteau font plutôt fonction d' « objet-preuve $»^{34}$ si cher à la narration fantastique.

Cahus disparaît du roman sans livrer la clef de l'énigme. Mais la frontière entre le monde rêvé et le monde réel ne s'en trouve pas restaurée pour autant, tant s'en faut: en revenant de la chapelle Saint-Augustin, le roi Arthur est attaqué par un démoniaque Chevalier Noir à la lance ardente, qui n'est autre que l'Homme Noir du rêve de Cahus ${ }^{35}$. Vaincu par le roi - à qui un mystérieux lien du sang l'unit, puisque sa lance ne peut être éteinte que dans le sang d'Arthur, et la blessure du roi, à son tour, ne peut être guérie qu'avec le sang de sa tête coupée -, il est ensuite dépiécé par une troupe de chevaliers qui emportent chacun une partie de son corps (et ce n'est là qu'un prélude à de nombreuses scènes de démembrements et décapitations dans le roman). Or, qu'un même personnage apparaisse dans le rêve et en réalité - réalité assez hallucinatoire, il faut l'admettre - l'on

30 V. A. Boureau, Satan hérétique: Histoire de la démonologie (1280-1330), Odile Jacob 2004, p. 190-192; idem, Satan et le dormeur : une construction de l'inconscient au Moyen Âge, « Chimères », 1991-1992, no. 14, p. 41-61.

31 Deux textes du XIV s. qui font allusion à l'aventure de Cahus, le roman de Fouke le Fitz Waryn et l'Historia de Jean de Glastonbury, ne précisent pas le côté ; pour Jean de Glastonbury il n'est même pas question du sommeil (v. M. Zink, op. cit. et W.A. Nitze, Le Haut Livre du Graal (Perlesvaus), vol. II, p. 108) .

32 F. Dubost, Aspects..., p. 790.

33 V. J.-Y. Tilliette, op. cit., p. 14, et B. Ramm, op. cit., p. 130

${ }^{34}$ L'expression est de J.-B.Baronian, Panorama de la littérature fantastique de langue française, Paris 1978, p. 68.

35 Et non pas son frère, comme le croit F. Dubost (Aspects..., op. cit., s. 792) ; le texte indique à mon avis clairement qu' il est le frère du chevalier mort (le chandelier d'or a bien été dérobé à celui-ci). 
peut en conclure que soit tout se passe en songe, soit le monde du rêve et celui de l'éveil sont perméables.

Ainsi, l'épisode de Cahus, en apparence superflu et extérieur par rapport à l'économie de la narration, devient une véritable clef des songes, ouvrant une perspective fantasmagorique où trouveront leur place images macabres de décollations, anthropophagie, créatures démoniaques et cruauté barbare, mélangées inextricablement aux visions lumineuses d'épiphanies célestes. Essayer d'en proposer à tout prix une senefiance ce serait à mon sens faire fausse route ; il vaut mieux se résoudre à accepter la « laide vérité » dans tout ce qu'elle a d'absurde : le paradoxe du rêve « faux » devenu « vrai » au défi du sens.

\section{Bibliographie}

Sources primaires:

Adélard de Bath, De eodem et diverso, éd. H. Willner, Münster 1903.

Saint Augustin, De Genesi ad litteram, PL 34.

Saint Augustin, De quantiate animae, PL 32.

Saint Augustin, La cité de Dieu, livres XV-XVIII, trad. G. Combès, Paris 1960.

Saint Augustin, Euvres complètes, t. VII, Paris 1873.

Saint Augustin, Sermo CCCVIII, PL 38.

Burchard de Worms, Corrector sive medicus [dans :] C. Vogel, Le pécheur et la pénitence au Moyen Âge, Paris 1969.

Grégoire le Grand, Dialogues, PL 77.

Honoré d'Autun, Elucidarium sive dialogus de summa totius christianae theologiae, PL 172.

Le Haut Livre du Graal (Perlesvaus), ed. W.A. Nitze, Th.A.Jenkins, New York 1972.

Le Haut Livre du Graal (Perlesvaus), ed. A. Strubel, Paris 2007.

Saint Jérôme, Ad Eustochium, PL 22.

Macrobe, Commenaire au Songe de Scipion, livre I, éd. M. Armisen-Marchetti, Paris 2001.

Reginon de Prüm, De ecclesiasticis disciplinis PL 132, col. 352B-352C

Tertullien, De anima, trad. E.-A. de Genoude, Paris 1852.

Sources secondaires :

Baronian J.-B., Panorama de la littérature fantastique de langue française, Paris 1978.

Boureau A., Satan et le dormeur : une construction de l'inconscient au Moyen Âge, « Chimères », 1991-1992, no. 14.

Boureau A., Satan hérétique: Histoire de la démonologie (1280-1330), Odile Jacob 2004.

Braet H., Le songe dans la chanson de geste, « Romanica Gandensia » 1975, no. 15. 
Dubost F., Aspects fantastiques de la littérature narrative médiévale (XII ${ }^{e}-\mathrm{XIII}{ }^{e}$ siècles). L'Autre, l'Ailleurs, l'Autrefois, Paris 1991.

Dubost F., Le Perlesvaus, livre de haute violence, « Senefiance » 1994, no. 36.

Gorecka-Kalita J., Les diaboliques du Perlesvaus ou les fantasmes de castration, « Romanica Cracoviensia » 2015, no. 15.

Krappe A.H., Balor with the Evil Eye, Studies in Celtic and French Literature, New York 1927.

Le Goff J., Le christianisme et les rêves [dans :] L’imaginaire médiéval, Paris 1985.

Maury A., Des hallucinations hypnagogiques ou des erreurs des sens dans l'état intermédiaire entre la veille et le sommeil, "Annales médico-psychologiques » 1848, I.

Méla Ch., La reine et le Graal. La conjointure dans les romans du Graal, de Chrétien de Troyes au Livre de Lancelot, Paris 1984.

Nitze W.A., Le Haut Livre du Graal (Perlesvaus), vol. II. (Commentary and Notes), New York 1972.

Ramm B., A Discourse for the Holy Grail in Old French Romance, Cambridge 2007.

Tilliette J.-Y., Belles-lettres et mauvais rêves [dans :] Le rêve médiéval, éd. A. Corbellari, J.-Y. Tillette, Genève 2007.

Valette J.-R., Barbarie et fantasmagorie dans Perlesvaus, le Haut Livre du Graal [dans :] Mélanges barbares. Hommage à Pierre Michel, éd. J.-Y. Debreuille, Ph. Régnier, Lyon 2001.

Verdon J., Dormir au Moyen Âge, [dans :] Revue belge de philologie et d'histoire, t. 72 , f. 4, 1994.

Verdon J., La nuit au Moyen Âge, Paris 1998.

Vogel C., Le pécheur et la pénitence au Moyen Âge, Paris 1969.

Williams A.M., Dreams and Visions in the Perlesvaus [dans :] B. Wheeler, Arthurian Studies in Honour of P.J.C. Field, Cambridge 2004.

Williamson M., The Dream of Cahus in the " Perlesvaus », " Modern Philology » 1932, Aug., vol. 30, no. 1.

Zink M., Le rêve avéré. La mort de Cahus et la langueur d'Arthur du Perlesvaus à Fouke le Fitz Waryn [dans :] Mélanges René Fromilhague, éd. Cl. Sicard, Université de Toulouse-Le Mirail, Toulouse 1984. 